\title{
Design paper of the "Blood pressure targets in post-resuscitation care and bedside monitoring of cerebral energy state: a randomized clinical trial"
}

Simon Mölström ${ }^{1 *}$ (D), Troels Halfeld Nielsen², Carl H. Nordström², Christian Hassager ${ }^{5}$, Jacob Eifer Møller ${ }^{3}$, Jesper Kjærgaard ${ }^{5}$, Sören Möller ${ }^{4}$, Henrik Schmidt ${ }^{1}$ and Palle Toft $^{1}$

\begin{abstract}
Background: Neurological injuries remain the leading cause of death in comatose patients resuscitated from out-of-hospital cardiac arrest (OHCA). Adequate blood pressure is of paramount importance to optimize cerebral perfusion and to minimize secondary brain injury. Markers measuring global cerebral ischemia caused by cardiac arrest and consecutive resuscitation and reflecting the metabolic variations after successful resuscitation are needed to assist a more individualized post-resuscitation care. Currently, no technique is available for bedside evaluation of global cerebral energy state, and until now blood pressure targets have been based on limited clinical evidence. Recent experimental and clinical studies indicate that it might be possible to evaluate cerebral oxidative metabolism from measuring the lactate-to-pyruvate (LP) ratio of the draining venous blood. In this study, jugular bulb microdialysis and immediate bedside biochemical analysis are introduced as new diagnostic tools to evaluate the effect of higher mean arterial blood pressure on global cerebral metabolism and the degree of cellular damage after OHCA.
\end{abstract}

Methods/design: This is a single-center, randomized, double-blinded, superiority trial. Sixty unconscious patients with sustained return of spontaneous circulation after OHCA will be randomly assigned in a one-to-one fashion to low (63 mm $\mathrm{Hg}$ ) or high $(77 \mathrm{~mm} \mathrm{Hg}$ ) mean arterial blood pressure target. The primary end-point will be a difference in mean LP ratio within $48 \mathrm{~h}$ between blood pressure groups. Secondary end-points are (1) association between LP ratio and all-cause intensive care unit (ICU) mortality and (2) association between LP ratio and survival to hospital discharge with poor neurological function.

Discussion: Markers measuring cerebral ischemia caused by cardiac arrest and consecutive resuscitation and reflecting the metabolic changes after successful resuscitation are urgently needed to enable a more personalized post-resuscitation care and prognostication. Jugular bulb microdialysis may provide a reliable global estimate of cerebral metabolic state and can be implemented as an entirely new and less invasive diagnostic tool for ICU patients after OHCA and has implications for early prognosis and treatment.

Trial registration: ClinicalTrials.gov (ClinicalTrials.gov Identifier: NCT03095742). Registered March 30, 2017.

Keywords: Out-of-hospital cardiac arrest, Neuroprotection, Blood pressure, Microdialysis, Cerebral metabolism

\footnotetext{
*Correspondence: simon.molstrom@rsyd.dk

1 Department of Anesthesiology and Intensive Care, Odense University

Hospital, J. B. Winsløws Vej 4, 5000 Odense C, Denmark

Full list of author information is available at the end of the article
}

(c) The Author(s). 2019 Open Access This article is distributed under the terms of the Creative Commons Attribution 4.0 International License (http://creativecommons.org/licenses/by/4.0/), which permits unrestricted use, distribution, and reproduction in any medium, provided you give appropriate credit to the original author(s) and the source, provide a link to the Creative Commons license, and indicate if changes were made. The Creative Commons Public Domain Dedication waiver (http://creativecommons.org/publicdomain/zero/1.0/) applies to the data made available in this article, unless otherwise stated. 


\section{Background}

In comatose patients resuscitated from out-of-hospital cardiac arrest $(\mathrm{OHCA})$, anoxic brain injuries remain the leading cause of death [1]. The overall mortality, though improved considerably over the last decade, continues to be significant and in most countries is as high as $90 \%[2,3]$. Despite successful resuscitation and admission to an intensive care unit (ICU), the in-hospital mortality is reported to be $30-50 \%[4,5]$. Functional favorable outcomes among OHCA survivors remain low, and global anoxic brain injury represents a major mechanism of long-term disability [6, 7]. During the post-resuscitative phase, the most significant clinical challenge is to limit secondary brain injury. Targeted temperature management (TTM) targeting $33-36{ }^{\circ} \mathrm{C}$ may mitigate cerebral ischemia-reperfusion injury and is recommended in current international guidelines [8-10]. However, managing post-cardiac arrest patients is far more complex than TTM alone, and brain-directed therapies include maintenance of normal oxygenation, ventilation, hemodynamic support to optimize cerebral perfusion, and glycemic control [10, 11].

Reduced cerebral blood flow $(\mathrm{CBF})$, which is mainly dependent upon mean arterial blood pressure (MAP), can result in brain ischemia and enhance secondary brain injury after cardiac arrest. Arterial hypotension may exacerbate brain injury following cardiac arrest because of ongoing cerebral hypoperfusion. Cerebral autoregulation is frequently impaired after cardiac arrest $[12,13]$, and brain perfusion declines when the MAP falls below 80 to $100 \mathrm{~mm} \mathrm{Hg}$. Thus, the generally recommended MAP of above $65 \mathrm{~mm} \mathrm{Hg}$ [11] to reverse the acute shock state may be inadequate to maintain adequate cerebral perfusion.

When considering blood pressure goals, clinicians should balance the metabolic needs of an ischemic brain with the potential for overstressing a decompensated heart. Post-cardiac arrest myocardial dysfunction, caused by coronary infarction and ischemia-reperfusion injury, is common after CA and resuscitation. Excessive vasopressor use may cause increased afterload and augmented oxygen consumption of the heart, thereby aggravating the myocardial damage [14]. High-dose vasopressor use has been associated with increased mortality [15].

Randomized control trials addressing specific MAP targets in post-resuscitation care have not yet been performed. This study addresses strategies for neuroprotection and will randomly assign a total of 60 unconscious resuscitated patients after OHCA in a double-blinded one-to-one fashion to low (mean $63 \mathrm{~mm} \mathrm{Hg}$ ) or high (mean $77 \mathrm{~mm} \mathrm{Hg}$ ) MAP target.

Secondary brain injury processes throughout the post-resuscitation period remain complex and involve numerous pathophysiological pathways that result from secondary ischemia and reperfusion injury. These include cerebral edema, inflammation, unbalanced CBF, and mitochondrial dysfunction [16]. Markers measuring global cerebral ischemia and reflecting the metabolic variations after resuscitation are needed for a more individualized post-resuscitation care and target-driven therapy to improve patient outcome $[17,18]$.

Microdialysis (MD) allows biochemical variables of the extracellular interstitial fluid to be monitored continuously and provides data on substrate supply and metabolism at the cellular level in the brain [19]. Cerebral energy metabolism is strictly aerobic, and the parameters monitored (glucose, pyruvate, lactate, glutamate, and glycerol) are closely linked (Fig. 1) [20]. Under clinical conditions, the cerebral cytoplasmic redox state is conventionally evaluated from the lactate-to-pyruvate (LP) ratio obtained from intracerebral MD and bedside biochemical analysis. Compromised energy metabolism will cause a shift in the cytoplasmic redox state that is immediately reflected in an increase of the LP ratio [21].

Clinical MD monitors the supply of glucose and its metabolism via glycolysis to pyruvate, which under oxidative conditions enter the tricarboxylic acid cycle. During hypoxic conditions or if mitochondrial function is compromised, pyruvate is metabolized to lactate. Therefore, the LP ratio is used as a marker of aerobic versus "anaerobic" metabolism not requiring oxygen [22]. High LP ratio is considered a robust indicator of anaerobic metabolism and the redox status of the tissue and is an independent predictor of mortality and unfavorable outcome in traumatic brain injury among patients monitored with MD [23-27]. An increased LP ratio could result from a failure of oxygen delivery (ischemic hypoxia) or no ischemic causes (e.g., mitochondrial dysfunction) [28]. An increase in the LP ratio in the presence of low pyruvate (and low oxygen pressure) indicates ischemia. In contrast, an increase in LP ratio in the presence of normal or high pyruvate (and normal oxygen pressure) indicates mitochondrial dysfunction. With intracerebral MD, the normal upper limit for the LP ratio of the human brain has been defined as 30 [22, 24]. Increased levels of glutamate and glycerol indicate hypoxia/ischemia and have been defined as indicators of excitotoxicity and cell membrane breakdown, respectively [19].

The LP ratio obtained from MD of cerebral venous blood may be a sensitive indicator of impending cerebral damage and secondary neurological deterioration and might play a critical role in detecting the early responses of post-resuscitation care. The use of global cerebral MD may potentially aid clinicians in providing 


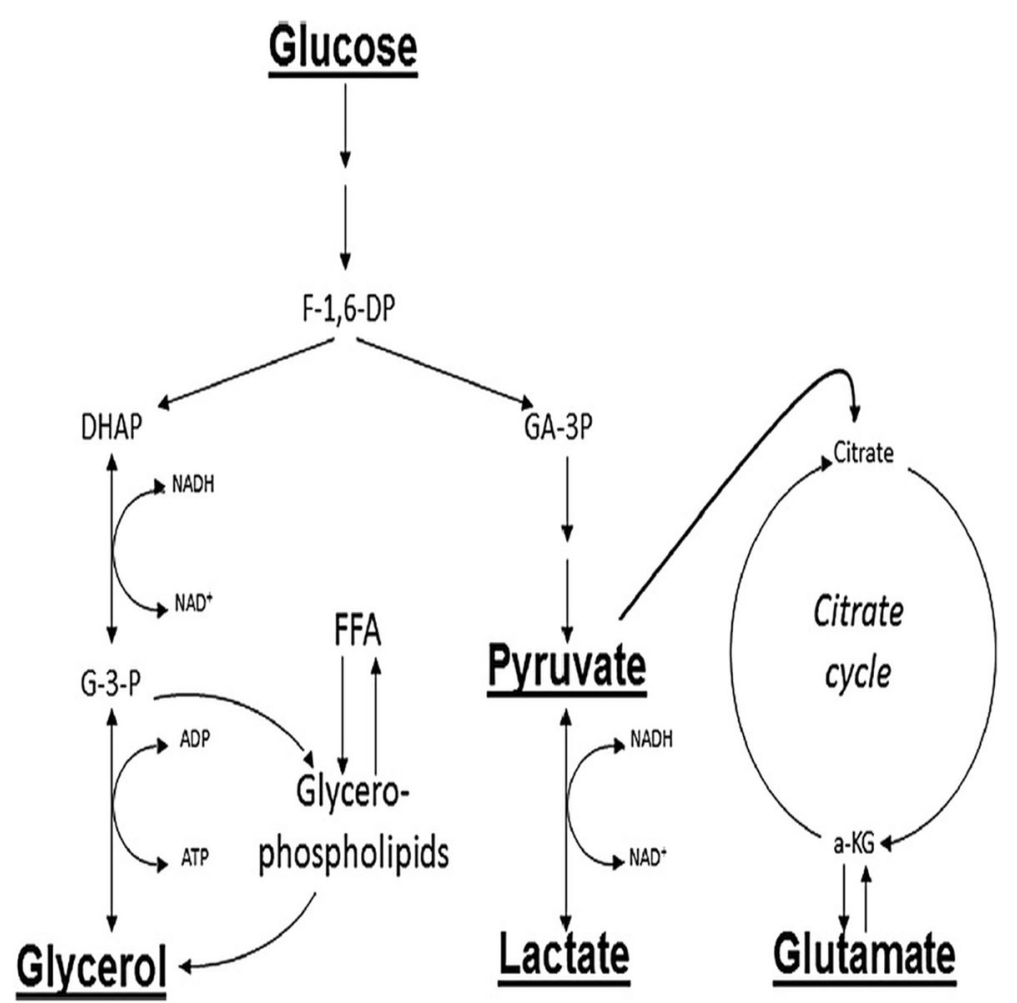

Fig. 1 Schematic diagram of cerebral metabolism. Schematic illustration of cerebral intermediary metabolism is shown with a focus on the glycolytic pathway and its relation to glycerol, glycerophospholipids, and the citric acid cycle. Abbreviations: a-KG a-ketoglutarate, DHAP dihydroxyacetone-phosphate, F-1,6-DP fructose-1,6-diphosphate, FFA free fatty acid, G-3-P glycerol-3-phosphate, GA-3P glyceraldehyde-3-phosphate. Underscored metabolites are obtained at the bedside with enzymatic techniques.

individualized and tailored brain resuscitation strategies that prevent secondary brain injury and lead to improved survival and neurological outcomes after cardiac arrest.

Our group recently reported how it is technically simple and feasible to place an MD catheter in the jugular bulb and monitor biochemical variables related to global cerebral energy metabolism at the bedside during cardiac surgery [29]. In the study, we evaluated a new method of cerebral metabolic monitoring, namely MD of cerebral venous blood drainage. The cerebral venous blood drains mainly to the jugular bulbs. Most of the outflow is to either the left or right side, depending on dominance. The study concluded that metabolic monitoring in the central venous outflow (jugular bulb) is representative of the overall cerebral metabolism and can be used in the diagnosis of compromised global cerebral metabolism during cardiac surgery.

\section{Methods/Design}

This study is a double-blinded, randomized, superiority clinical trial assessing the effect of different blood pressure levels on global cerebral metabolism in addition to TTM in adult comatose patients resuscitated from OHCA. Patients are enrolled at one Danish university hospital with a population background of about 1.3 million citizens for highly specialized cardiac care. The SPIRIT (Standard Protocol Items: Recommendations for Interventional Trials) 2013 Checklist, together with the Statement, facilitated with drafting of the trial protocol (Additional file 1).

\section{Aim}

The study aims are to (a) investigate whether the LP ratio obtained by $\mathrm{MD}$ of the cerebral venous outflow reflects a derangement of global cerebral energy state after OHCA, (b) investigate the effect of higher MAP on global cerebral metabolism and LP ratio in comatose patients resuscitated from OHCA, and (c) investigate the correlation between $\mathrm{LP}$ ratio and neurological outcome (cerebral performance category, or $\mathrm{CPC}$ ). The null hypothesis is that a higher MAP will not reduce LP ratio within $48 \mathrm{~h}$ compared with lower MAP. 


\section{Trial procedure}

\section{Phase 1 (hospital admission to intervention onset)}

Comatose patients after OHCA admitted to the hospital with sustained return of spontaneous circulation (ROSC) are eligible for screening. Acute computed tomography (CT) scan or acute coronary angiogram performed before ICU admission is also included in this phase. The inclusion window is $220 \mathrm{~min}$ from ROSC to screening. Patients eligible for the trial must comply with all inclusion criteria according to Table 1 at randomization. In accordance with Danish legislation, if no exclusion criteria are met, proxy informed consent is obtained from next of kin and from one independent medical doctor not involved with the trial.

Enrollment of patients will be performed by the attending physician who is not involved in data collection

Table 1 Inclusion and exclusion criteria

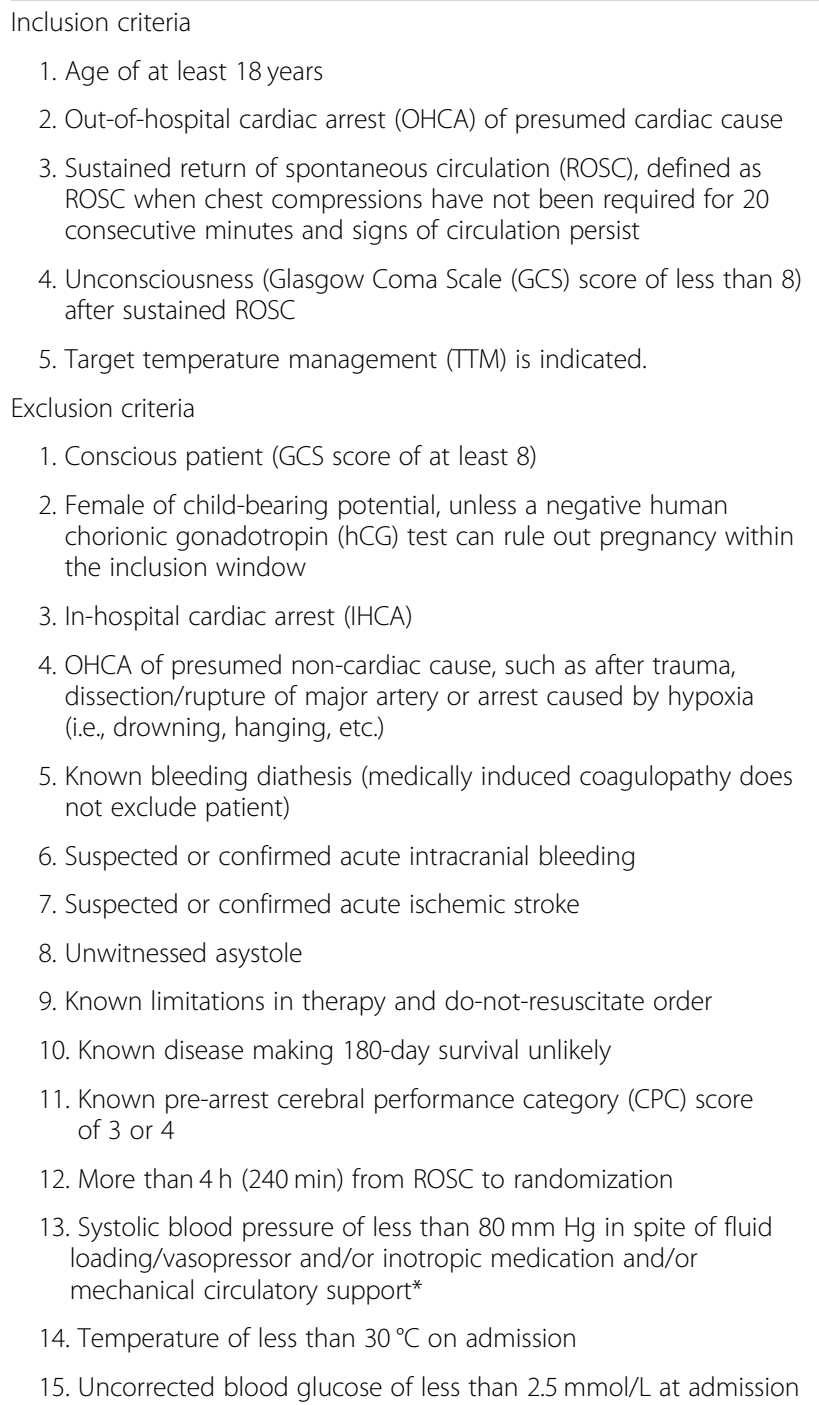

* If systolic blood pressure is recovering during the inclusion window the patient can be included or assessment. Randomization of blood pressure target was performed by a computerized algorithm using STATA (StataCorp LLC, College Station, TX, USA) in varying block sizes of 4,8 , and 12 patients. Baseline characteristics are obtained.

\section{Phase 2 (intervention period)}

OHCA patients are randomly allocated to either lowor high-blood pressure target during ICU stay: Allocation of patients resuscitated from OHCA to low- or high-blood pressure target during ICU stay):. The first invasive blood pressure using the trial blood pressure module is marking the start of the intervention, most often coinciding with initiation of TTM. Interventions are considered emergency procedures, and study blood pressure measurement using the study blood pressure modules (HP/Philips M1006B Invasive Pressure module) is commenced as soon as possible after sustained ROSC, screening, and randomization. Study target blood pressure will be blinded. In half of the modules, the calibration factor will be adjusted in order to show the blood pressure measurements about $10 \%$ lower than the patients' actual blood pressure at $100 \mathrm{~mm} \mathrm{Hg}$, and in half of the patients, the blood pressure measurements will be shown to be about $10 \%$ higher at $100 \mathrm{~mm} \mathrm{Hg}$. Targeting an MAP of $70 \mathrm{~mm} \mathrm{Hg}$ during treatment in both groups will mean a blinded comparison of 63 and $77 \mathrm{~mm} \mathrm{Hg}$, both of which are within the usually acceptable ranges of blood pressure. A prospective, randomized, controlled clinical study has validated this method for double-blinded comparison of MAP targets in the ICU setting [30]. The intervention continues for as long as the patient has invasive blood pressure measurements.

Blood pressure management during ICU stay MAP is increased by administration of (a) volume resuscitation until CVP is at least $10 \mathrm{~mm} \mathrm{Hg}$ and distensibility index of the inferior vena cava is less than $12 \%$, unless pulmonary edema is clinically apparent, and (b) norepinephrine $(\mu \mathrm{g} / \mathrm{kg}$ per min solution) is administered in increments of $0.02 \mu \mathrm{g} / \mathrm{kg}$ per min until a dose of $0.2 \mu \mathrm{g} /$ $\mathrm{kg}$ per min is reached. A higher dose will be used at the discretion of the attending physician; (c) dopamine ( $\mu \mathrm{g} /$ $\mathrm{kg}$ per min solution) will be used in addition to norepinephrine at a maximal dose of $10 \mu \mathrm{g} / \mathrm{kg}$ per min.

As part of the routine hemodynamic monitoring, a pulmonary artery catheter (PAC) is inserted via the internal jugular or subclavian vein under ultrasound guidance. During ICU stay, cardiac index, mixed venous oxygen saturation $\left(\mathrm{SvO}_{2}\right)$, central venous, and pulmonary pressures were measured continuously by PAC (CCOmbo PAC ${ }^{\circ}$ Edwards Lifesciences, Irvine, CA, USA) linked to the correct monitor (Vigilance II, Edwards Lifesciences). These data, together with 
information on blood temperature, oxygen saturation, and MAP measured from a radial artery line, were transferred electronically to a computer with a 2 -second time interval. If echocardiography, and thermodilution suggest that low cardiac output addition of an inotropic agent may be appropriate). The following agents may be considered: (a) milrinone (0.375$0.75 \mu \mathrm{g} / \mathrm{kg}$ per min) and (b) levosimendan $(0.1 \mu \mathrm{g} / \mathrm{kg}$ per min). If an inotropic agent at maximal dose combined with vasopressors cannot maintain cardiac output, mechanical circulatory support may be considered.

Neuromonitoring during ICU stay At ICU arrival, patients will be implanted with a jugular bulb MD catheter and monitored for $96 \mathrm{~h}$ or until arousal. MD samples are collected in microvials and analyzed every $1 \mathrm{~h}$ at the bedside. Regional cerebral oxygen saturation will be monitored by using bifrontal NIRS (Somanetics INVOS Cerebral Oximeter system). Right and left frontal regional oxygen saturation $\left(\mathrm{rSO}_{2}\right)$ values will be recorded simultaneously during ICU stay, and values will be recorded every hour for $96 \mathrm{~h}$ or until arousal.

\section{Mechanical ventilation and oxygenation during ICU}

stay Patients will be mechanically ventilated, sedated, and when necessary paralyzed with neuromuscular blocking agents to reduce shivering and subsequent heat generation and energy consumption. The study is targeting normal oxygenation (13-14 $\mathrm{kPa})$ during TTM and mechanical ventilation. Ventilation is adjusted, targeting normocapnia of partial pressure of carbon dioxide $\left(\mathrm{paCO}_{2}\right)$ of 4.5-6.0 in all patients.

Serial arterial blood gas will be analyzed open-label by using commercially available equipment adjusted to $37^{\circ} \mathrm{C}$ (alpha-stat). The TTM intervention period of $24 \mathrm{~h}$ commenced at the time of randomization by using an automated feedback device with temperature control to achieve a target core temperature of $36^{\circ} \mathrm{C}$. After TTM, patients are rewarmed to a core temperature of $37^{\circ} \mathrm{C}$ with no more than $0.5^{\circ} \mathrm{C}$ per hour. Phase 2 ends when sedation is withheld.

\section{Phase 3 (from end of sedation to $72 \mathrm{~h}$ after OHCA)}

Sedation is terminated after rewarming when the temperature is at least at $37^{\circ} \mathrm{C}$. Normothermia of $37^{\circ} \mathrm{C}$ $\pm 0.5^{\circ} \mathrm{C}$ is maintained until $72 \mathrm{~h}$ after the cardiac arrest as long as the patients are managed in the ICU and are unconscious. Nevertheless, weaning from ventilation will be attempted at the earliest possible time during this phase on the basis of typical procedures for discontinuation of mechanical ventilation. Blinded physicians perform a neurological evaluation of patients who remain in coma at $72 \mathrm{~h}$ or later after OHCA. The number of patients still comatose at $72 \mathrm{~h}$ after the end of
TTM who underwent neurological prognostication will be reported. The number of patients who died before neurological prognostication and their presumed cause of death, including limitations in care and explanations for that, will be described. If judged by attending physicians to be indicated, electroencephalogram, somatosensory evoked potentials, and $\mathrm{CT}$ of the brain will be performed.

\section{Phase 4 (end of intervention period to hospital discharge)}

This starts when invasive blood pressure measurement is discontinued during the ICU stay.

Neurological outcome will be assessed at hospital discharge in accordance with the CPC scale [31, 32]: CPC 1 , no neurological deficit; CPC 2, mild to moderate dysfunction; CPC 3, severe dysfunction; CPC 4, coma; and CPC 5, death. CPC scores of 1 and 2 are mostly considered "good" outcomes, and a CPC 3-4 "poor" outcomes. Physicians performing the CPC assessment will be blinded to patient's LP ratio levels.

\section{Phase 5 (hospital discharge/ 3 months after end of trial)} Vital status and neurological status (CPC) were evaluated at 3 months after admission by clinical examination or telephone interview. Vital status will be evaluated at the end of the trial by using the Danish civil registration system. The SPIRIT timeline of progress through the phases of the parallel-randomized trial is shown in Fig. 2.

\section{Inclusion}

Patients at least 18 years old with sustained ROSC after $\mathrm{OHCA}$ are eligible for inclusion if complying with all the criteria presented in Table 1.

\section{Bedside monitoring of cerebral energy microdialysis}

Patients will be implanted with a jugular bulb MD catheter (CMA 67 IV $130 \mathrm{~mm}$, MDialysis AB, Stockholm, Sweden) and monitored for $96 \mathrm{~h}$ or until arousal. The catheter allows energy-related metabolites to diffuse into the catheter reflecting accurate concentrations in the venous blood, $\mathrm{CE}$ marked according to the Medical Device Directive, 93/42/EEC. MD catheters are inserted in a retrograde direction into the jugular bulb, through a peripheral intravenous $17 \mathrm{GA}$ cannula by using ultrasound guidance, as performed in a previous study [29]. The positioning of the catheter tip in the jugular bulb above the inlet of the common facial vein is confirmed on cranial CT scan in accordance with earlier practice [33]. MD pumps (CMA 106, MDialysis AB) at $0.3 \mu \mathrm{L} / \mathrm{min}$ perfuse $\mathrm{MD}$ catheters. The perfusates are collected in microvials, analyzed every $1 \mathrm{~h}$ for minimum $96 \mathrm{~h}$ by enzymatic photometric techniques, and presented at the bedside (Iscus, Mdialysis AB). 


\begin{tabular}{|c|c|c|c|c|c|c|c|c|}
\hline \multirow[b]{3}{*}{ TIMEPOINT } & \multicolumn{8}{|c|}{ STUDY PERIOD } \\
\hline & \multirow{2}{*}{$\frac{\text { Enrolment }}{\text { HA }}$} & \multirow{2}{*}{$\frac{\text { Allocation }}{0}$} & \multicolumn{5}{|c|}{ Post-allocation } & \multirow{2}{*}{$\frac{\text { Close-out }}{3 \text { months }}$} \\
\hline & & & 24 & 48 & 72 & 96 & HD & \\
\hline \multicolumn{9}{|l|}{ ENROLMENT: } \\
\hline \multirow{2}{*}{$\begin{array}{l}\text { Eligibility screen } \\
\text { Informed consent }\end{array}$} & $x$ & & & & & & & \\
\hline & $x$ & & & & & & & \\
\hline Allocation & & $x$ & & & & & & \\
\hline \multicolumn{9}{|l|}{ INTERVENTIONS: } \\
\hline \multicolumn{9}{|l|}{ LoW MAP } \\
\hline \multicolumn{9}{|l|}{ High MAP } \\
\hline \multicolumn{9}{|l|}{ TTM } \\
\hline \multicolumn{9}{|l|}{ ASSESSMENTS: } \\
\hline Microdialysis & $x$ & $x$ & $x$ & $x$ & $X$ & $x$ & & \\
\hline NIRS & & $x$ & $x$ & $x$ & $X$ & $x$ & & \\
\hline PAC & & $x$ & $X$ & $x$ & $X$ & $x$ & & \\
\hline CPC & & & & & & & $X$ & $X$ \\
\hline $\begin{array}{l}\text { Neurological } \\
\text { prognostication }\end{array}$ & & & & & $X$ & $X$ & & \\
\hline
\end{tabular}

Fig. 2 Overall schedule and time commitment for trial participants. Abbreviations: CPC cerebral performance category, HA hospital admission, HD hospital discharge, MAP mean arterial blood pressure, NIRS near infrared spectroscopy, PAC pulmonary artery catheter, TTM targeted temperature management.

The analyses include the biochemical variables regularly monitored during intracerebral MD: glucose, pyruvate, lactate, glutamate, and glycerol. Clinical data, radiology, electroencephalography, somatosensory evoked potential, and NIRS will be recorded.

\section{Logistics}

The study is enrolling at one university hospital with experience in conducting clinical trials. All investigators have been qualified and are licensed in "good clinical practice" (GCP).

\section{Patient and public involvement}

Patients were not involved in setting the research hypothesis or end-points, nor were they implicated in the design of the study. There are no plans to include patients in the dissemination of results, nor will we publish results directly to patients.

\section{End-points}

The study focuses on the following end-points:

Primary end-point

1. Comparing mean LP ratio differences within $48 \mathrm{~h}$ between blood pressure groups and comparing individual LP ratio measurements using longitudinal analysis.

Secondary end-points

1. Association between LP ratio and all-cause mortality during ICU stay adjusted for blood pressure groups

2. Between-group difference in total duration (minutes) of cerebral desaturation defined as an absolute $\mathrm{rSO}_{2}$ of not more than $50 \%$ during ICU stay

3. Between-group difference within $48 \mathrm{~h}$ in lactate, pyruvate, glutamate, glycerol, and glucose measured in jugular bulb 
4. Association between LP ratio and cardiac index adjusted for blood pressure groups

5. Association between LP ratio and survival to hospital discharge with poor neurological function (CPC 3-4) adjusted for blood pressure groups.

\section{Sample size estimation}

The sample size was calculated on the basis of the primary hypothesis but taking into account only the mean LP ratio and not the individual measurements; this was due to insufficient information about interpretation variation in LP values; this implies a conservative error and hence the true power of the study is expected to be higher than in this calculation. The trial is designed as a randomized study, and we have chosen to power this study according to differences in the LP ratio. A difference of $30 \%$ between groups is defined as the minimal clinically relevant difference. Assuming an average LP ratio of less than 20 over $48 \mathrm{~h}$ in the high MAP group compared with more than 35 over $48 \mathrm{~h}$ in the low MAP group, we will need to include 46 patients in total to achieve a power of 0.9 using a patient-to-patient variation with standard deviation (SD) of 15 in LP ratio as motivated by an earlier study [29] in the power calculation and assuming normal distribution of LP ratio. To take possible higher patient-to-patient variation in this study as well as deviations from normality into account, we plan to include at least 60 patients in total. Defining an LP ratio of more than 30 averaged over 24 and $48 \mathrm{~h}$ for both groups, we will analyze the proportion of patients reaching that end-point in each group.

\section{Statistical analysis plan}

The statistical analyses will be the following:

1. Analyses will be performed in accordance with the intention-to-treat principle and will take into account patients lost to follow-up.

2. A two-sided significance level of 0.05 will be applied to both primary and secondary end-points.

3. Unpaired $t$ tests or Mann-Whitney $U$ tests will be conducted for unpaired comparisons of numerical variables. A chi-squared or Fisher's exact test will be conducted to examine differences between categorical variables.

4. Association between LP ratio and "poor" neurological outcome (CPC 3-4) will be assessed with chi-squared test and logistic regression. The overall expected rates of unfavorable neurological outcome (CPC 3-4) are $5-7 \%$, observed in earlier studies [34, 35]. The multivariate logistic model will be adjusted for time to ROSC, arterial carbon dioxide partial pressure, baseline LP, and average LP ratio $>30$ $>24$ and $48 \mathrm{~h}$ as covariates.
5. Association between LP ratio and all-cause mortality will be assessed with chi-squared test and logistic regression.

6. Receiver operating characteristic (ROC) curves will be constructed to determine the sensitivity and specificity of the LP ratio levels at each time point (and the maximal LP ratio level recorded during the ICU period) for predicting outcomes.

7. Association between total duration (in minutes) of cerebral desaturation $\left(\mathrm{rSO}_{2} \leq 50 \%\right)$ and unfavorable neurological outcome (CPC 3-4) and death will be assessed with chi-squared test and logistic regression.

8. Time from start of randomization to death in the two blood pressure groups will be assessed by using the Kaplan-Meier method, and group differences will be tested by log-rank test. Cox regression will be applied for adjusted comparisons and estimation of hazard ratios.

9. In regard to dynamic changes of MD variables in the two blood pressure groups, longitudinal models, applying linear mixed effects regression, will be used to account for repeated measurements of MD biomarkers across different patients over time.

10. In regard to dynamic changes of blood pressure, longitudinal models, applying linear mixed effects regression, will be used to account for repeated measurements of blood pressure across different patients over time.

All standard assumptions regarding statistical models will be checked. Statistical analysis will be performed by using STATA V.15 (StataCorp LLC).

\section{Trial profile}

A flowchart of the study participants will be presented in accordance with the Consolidated Standards of Reporting Trials (CONSORT) diagram [36, 37].

\section{Baseline data}

The predefined baseline variables are the following:

1. Sex

2. Age

3. Comorbidities (premorbid CPC, ischemic heart disease, heart failure, previous cardiac arrest, arterial hypertension, stroke, epilepsy, diabetes, chronic obstructive pulmonary disease, chronic hemodialysis, and alcoholism)

4. Previous percutaneous coronary intervention

5. Previous coronary artery bypass graft

6. Previous valvular surgery

7. Implantable cardioverter-defibrillator or pacemaker or both

8. Pre-hospital variables 
a. Location of cardiac arrest

b. Bystander-witnessed arrest

c. Bystander cardiopulmonary resuscitation

d. Shockable primary rhythm

e. Time to basic life support

f. Time to advanced life support

g. Time to ROSC

9. Admission variables
a. First measured temperature
b. Glasgow Coma Scale score
c. Shock at admission
d. Presumed cause of cardiac arrest (cardiac/ non-cardiac)
e. Acute myocardial infarction
f. Serum $\mathrm{pH}$
g. Serum lactate
h. End-tidal carbon dioxide
i. Blood pressure
j. Result of angiogram
k. Cardiac troponin (TnI) and creatine kinase- muscle/brain (CK-MB).

Differences in baseline variables between blood pressure groups will be analyzed and presented in tables. Continuous variables will be presented as mean $\pm \mathrm{SD}$, and differences will be analyzed with the unpaired $t$ test. In case of non-normally distributed data, continuous variables will be presented as median (interquartile range) and a $t$ test will be applied following logarithmic transformation if the transformed data are normally distributed; alternatively, the non-parametric Mann-Whitney test will be applied. Categorical variables will be presented as counts and percentages and differences will be analyzed with the chi-squared test or Fisher's exact tests if counts below 10 are observed.

\section{Discussion}

The mortality of patients who are admitted in a comatose state to an ICU following successful resuscitation after cardiac arrest remains significant. The necessity for early and accurate prognostic predictors is important, especially since sedation and TTM might change the neurological examination and postpone the recovery of motor response for several days. Markers measuring global cerebral ischemia caused by cardiac arrest and consecutive resuscitation and reflecting the metabolic variations after successful resuscitation are urgently required to assist more personalized post-resuscitation care and prognostication.

Jugular bulb MD may provide a reliable global estimate of cerebral metabolic state and can be implemented as an entirely new and less invasive diagnostic tool for ICU patients after OHCA and has implications for early prognosis and treatment. The LP ratio assessed from MD of cerebral venous blood may play a critical role in detecting the early responses of post-resuscitation care and may predict in-hospital and long-term prognosis in patients affected by brain injury after cardiac arrest. In the future, this might optimize and individualize the treatment of post-cardiac arrest patients and potentially improve outcome.

\section{Surrogate end-point}

The end-point defined as the LP ratio during ICU stay is a surrogate marker for poor neurological outcome and death. The surrogate marker is used as an alternative to a hard end-point in order to power this study adequately. Previously, LP ratio studies have been shown to be a reliable marker for poor outcome after traumatic brain injury [22-26]. Hence, our surrogate end-point reflects the clinically relevant question of whether higher MAP has a potential neuroprotective effect in our population.

\section{Strengths and limitations of this study}

- Jugular bulb MD may provide a reliable global estimate of cerebral metabolic state and can be implemented as an entirely new and less invasive diagnostic tool for ICU patients after OHCA and has implications for early prognosis and treatment.

- This pragmatic trial is addressing the effect of higher MAP on global cerebral metabolism in patients resuscitated from OHCA.

- Randomized controlled trial design minimizes the risk of selection bias.

- Clinicians are not blinded to data obtained at the bedside from jugular bulb MD, so all outcome assessors will be blinded to minimize the risk of bias.

- Until now, no established gold standard method assessing global cerebral energy state has existed. Therefore, it is not possible to compare jugular bulb MD with an established method already in clinical use.

\section{Trial status}

Protocol version 1.1. Date: March 8, 2018. The study is ongoing and currently enrolling. Recruitment began January 11, 2017, and is expected to be completed around January 8, 2020.

\section{Additional files}

Additional file 1: SPIRIT (Standard Protocol Items: Recommendations for Interventional Trials) 2013 Checklist: Recommended items to address in a clinical trial protocol and related documents*. (PDF $144 \mathrm{~kb}$ )

Additional file 2: Informed consent. (PDF $183 \mathrm{~kb}$ ) 


\section{Abbreviations}

CBF: Cerebral blood flow; CPC: Cerebral performance category; $\mathrm{CT}$ : Computed tomography; ICU: Intensive care unit; LP ratio: Lactate-topyruvate ratio; MAP: Mean arterial blood pressure; MD: Microdialysis; NIRS: Near infrared spectroscopy; OHCA: Out-of-hospital cardiac arrest; PAC: Pulmonary artery catheter; ROSC: Return of spontaneous circulation; $\mathrm{rSO}_{2}$ : Regional oxygen saturation; SD: Standard deviation; SPIRIT: Standard Protocol Items: Recommendations for Interventional Trials; TTM: Targeted temperature management

\section{Acknowledgments}

Not applicable.

\section{Funding}

Until now, the study has been supported by non-profit organizations, including the University of Southern Denmark, Region of Southern Denmark, and Department of Anesthesiology and Intensive Care, Odense. The study received a grant from the A.P. Møller Foundation for the Advancement of Medical Science of 50.000 DKK (grant number 17-L-0030). Any additional funding will be included along with the publication of trial results.

\section{Availability of data and materials}

Study data will be collected and managed by using Research Electronic Data Capture REDCap electronic data capture tools hosted at Odense Patient data Explorative Network. REDCap [38] is a secure, web-based application designed to support data capture for research studies, providing (1) an intuitive interface for validated data entry, (2) audit trails for tracking data manipulation and export procedures, (3) automated export procedures for seamless data downloads to common statistical packages, and (4) procedures for importing data from external sources. An external independent person is responsible for assigning randomization codes at the end of trial and thereby securing the actual blood pressure values for the database. All data will be anonymized before analyses. The Danish Data Protection Agency has approved the study. The datasets used or analyzed (or both) during the current study are available from the corresponding author on reasonable request

\section{Ethical justification}

Participation in the MICA trial will not interfere with or delay routine diagnostic or therapeutic procedures.

The ethical justifications for interventions in the MICA trial are the following: 1. Knowledge of effect of different blood pressures levels effect on cerebral metabolism in comatose patients resuscitated after OHCA cannot be acquired outside the acute setting. Research in a non-acute setting is not conceivable, and assessment in a human experimental model is clearly unethical. No translational models mimic the scenario of OHCA adequately. 2. The interventions should be commenced as soon as possible after ROSC since blood pressure targets may be of importance in neuroprotection via mechanisms thought to reduce reperfusion injuries in the brain. Therefore, consent from patients is not feasible.

3. Inclusion in the trial will provide knowledge that may benefit management of OHCA patients in general since further knowledge is needed to continue optimization of the neuroprotective interventions in the post-resuscitation phase.

\section{Publication plan and author policy}

The results of the trial will be published regardless of whether they are positive, negative, or inconclusive. The final manuscript will be submitted to a peer-reviewed international journal. Authorship will be granted by using the Vancouver definitions and depending on personal involvement.

\section{Authors' contributions}

THN, CHN, and JEM are investigators at Odense University Hospital and assisted with data analysis and drafting the main manuscript. $\mathrm{CH}$ is an investigator at Copenhagen University Hospital, sponsor, and initiator of the study and assisted with data analysis and drafting the main manuscript. JK is the principal investigator at Copenhagen University Hospital, sponsor, and initiator of the study and is responsible for funding as well as relevant approvals. SMöll is a biostatistician at OPEN (Odense Patient data Explorative Network) and carried out statistical analyses on clinical data and consulting on statistical methods. PT is an investigator at Odense University Hospital and assisted with data analysis and drafting the manuscript. HS is an investigator at Odense University Hospital and assisted with data analysis, logistics ensuring availability of trial blood pressure modules, education of staff in trial-related procedures, recruitment, follow-up of patients, and entry of data daily management and recruitment of patients. SMöls is the principal investigator at Odense University Hospital, sponsor, and initiator of the study and is responsible for funding as well as relevant approvals, coordination of protocol development, information, recruitment of patients, daily management, data analysis, and drafting of manuscripts. All authors read and approved the final manuscript

\section{Ethics approval and consent to participate}

The trial is conducted in accordance with national and international standards for good clinical practice. The local ethics committee has formally approved the protocol and any amendments, including written information and the consent forms prior to initiation of the trial (trial registration: S-20150173 HLP). The protocol is available at www.clinicaltrials.gov (ClinicalTrials.gov Identifier: NCT03095742). This trial will be conducted in accordance with national and international standards of good clinical practice. Eligible patients are by inclusion criteria unconscious and unable to give informed consent in the acute setting. Since blood pressure targets may be of importance in neuroprotection via mechanisms thought to reduce reperfusion injuries in the myocardium and in the brain, treatment allocation should be performed as soon as possible. Furthermore, our group has recently reported how it is technically simple and safe to insert a microdialysis catheter in the jugular bulb and monitor biochemical variables related to global cerebral energy metabolism at the bedside during cardiac surgery [29]. Therefore, randomization is performed immediately at admission before informed consent can be obtained according to the Declaration of Helsinki. Patients recovering consciousness will be requested to give informed consent. Legal representatives of the patients (an MD not participating in the study) will be informed and consent will be obtained as quickly as possible. In addition, next of kin will be provided with written and oral information on this trial, allowing them to make an informed decision about participation in this trial. The investigator uses an institutional review board-approved script (Additional file 2) that judiciously describes the nature of the intervention, including the use of jugular bulb microdialysis. The consent form must be signed by the participant or legally acceptable surrogate and by the investigator seeking the consent.

\section{Consent for publication}

Not applicable.

\section{Competing interests}

The authors declare that they have no competing interests.

\section{Publisher's Note}

Springer Nature remains neutral with regard to jurisdictional claims in published maps and institutional affiliations.

\section{Author details \\ ${ }^{1}$ Department of Anesthesiology and Intensive Care, Odense University Hospital, J. B. Winsløws Vej 4, 5000 Odense C, Denmark. ${ }^{2}$ Department of Neurosurgery, Odense University Hospital, J. B. Winsløws Vej 4, Odense 5000, Denmark. ${ }^{3}$ Department of Cardiology, Odense University Hospital, J. B. Winsløws Vej 4, Odense 5000, Denmark. ${ }^{4}$ OPEN - Odense Patient data Explorative Network, University of Southern Denmark, Odense University Hospital and Department of Clinical Research, J. B. Winsløws Vej 9, Odense 5000, Denmark. ${ }^{5}$ The Heart Centre, Copenhagen University Hospital, Blegdamsvej 9, Copenhagen 2100, Denmark.}

Received: 11 October 2018 Accepted: 6 May 2019 Published online: 10 June 2019

References

1. Laver S, Farrow C, Turner D, Nolan J. Mode of death after admission to an intensive care unit following cardiac arrest. Intensive Care Med. 2004;30:2126-8.

2. Herlitz J, Eek M, Engdahl J, Holmberg M, Holmberg S. Factors at resuscitation and outcome among patients suffering from out of hospital cardiac arrest in relation to age. Resuscitation. 2003;58:309-17.

3. Grasner JT, Lefering R, Koster RW, Masterson S, Bottiger BW, Herlitz J, et al. EuReCa ONE-27 Nations, ONE Europe, ONE Registry: A prospective one month analysis of out-of-hospital cardiac arrest outcomes in 27 countries in Europe. Resuscitation. 2016;105:188-95. 
4. Soholm H, Wachtell K, Nielsen SL, Bro-Jeppesen J, Pedersen F, Wanscher $\mathrm{M}$, et al. Tertiary centres have improved survival compared to other hospitals in the Copenhagen area after out-of-hospital cardiac arrest. Resuscitation. 2013;84:162-7.

5. Bro-Jeppesen J, Kjaergaard J, Horsted TI, Wanscher MC, Nielsen SL, Rasmussen $\mathrm{LS}$, et al. The impact of therapeutic hypothermia on neurological function and quality of life after cardiac arrest. Resuscitation. 2009;80:171-6.

6. Arawwawala D, Brett SJ. Clinical review: beyond immediate survival from resuscitation-long-term outcome considerations after cardiac arrest. Crit Care. 2007;11:235.

7. Koenig MA. Brain resuscitation and prognosis after cardiac arrest. Crit Care Clin. 2014;30:765-83.

8. Bernard SA, Gray TW, Buist MD, Jones BM, Silvester W, Gutteridge G, et al. Treatment of comatose survivors of out-of-hospital cardiac arrest with induced hypothermia. N Engl J Med. 2002;346:557-63.

9. Hypothermia after Cardiac Arrest Study G. Mild therapeutic hypothermia to improve the neurologic outcome after cardiac arrest. N Engl J Med. 2002;346:549-56.

10. Nolan JP, Soar J, Cariou A, Cronberg T, Moulaert VR, Deakin CD, et al. European Resuscitation Council and European Society of Intensive Care Medicine 2015 guidelines for post-resuscitation care. Intensive Care Med. 2015;41:2039-56.

11. Callaway CW, Donnino MW, Fink EL, Geocadin RG, Golan E, Kern KB, et al. Part 8: Post-Cardiac Arrest Care: 2015 American Heart Association Guidelines Update for Cardiopulmonary Resuscitation and Emergency Cardiovascular Care. Circulation. 2015;132(18 Suppl 2):S465-82.

12. Nishizawa $\mathrm{H}$, Kudoh I. Cerebral autoregulation is impaired in patients resuscitated after cardiac arrest. Acta Anaesthesiol Scand. 1996:40:1149-53.

13. Sundgreen C, Larsen FS, Herzog TM, Knudsen GM, Boesgaard S, Aldershvile J. Autoregulation of cerebral blood flow in patients resuscitated from cardiac arrest. Stroke. 2001;32:128-32.

14. Jakkula P, Pettila V, Skrifvars MB, Hastbacka J, Loisa P, Tiainen M, et al. Targeting low-normal or high-normal mean arterial pressure after cardiac arrest and resuscitation: a randomised pilot trial. Intensive Care Med. 2018;44:2091-101.

15. Bro-Jeppesen J, Annborn M, Hassager C, Wise MP, Pelosi P, Nielsen N, et al. Hemodynamics and vasopressor support during targeted temperature management at 33 degrees $C$ Versus 36 degrees $C$ after out-of-hospital cardiac arrest: a post hoc study of the target temperature management trial*. Crit Care Med. 2015;43:318-27.

16. Sinha N, Parnia S. Monitoring the Brain After Cardiac Arrest: a New Era. Curr Neurol Neurosci Rep. 2017;17:62.

17. Nordstrom $\mathrm{CH}$, Nielsen $\mathrm{TH}$. Continuous determination of optimal cerebral perfusion pressure--in search of the Holy Grail? Crit Care Med. 2013;41:e4-5.

18. Steiner LA, Czosnyka M, Piechnik SK, Smielewski P, Chatfield D, Menon DK, et al. Continuous monitoring of cerebrovascular pressure reactivity allows determination of optimal cerebral perfusion pressure in patients with traumatic brain injury. Crit Care Med. 2002;30:733-8.

19. Hutchinson PJ, Jalloh I, Helmy A, Carpenter KL, Rostami E, Bellander BM, et al. Consensus statement from the 2014 International Microdialysis Forum. Intensive Care Med. 2015:41:1517-28.

20. Nordstrom CH. Cerebral microdialysis in TBI-limitations and possibilities. Acta Neurochir. 2017:159:2275-7.

21. Siesjö BK. Brain energy metabolism and catecholaminergic activity in hypoxia, hypercapnia and ischemia. J Neural Transm Suppl. 1978;(14):17-22.

22. Hutchinson P, O'Phelan K, Participants in the International Multidisciplinary Consensus Conference on Multimodality M. International multidisciplinary consensus conference on multimodality monitoring: cerebral metabolism. Neurocrit Care. 2014;21(Suppl 2):S148-58.

23. Vespa PM, Miller C, McArthur D, Eliseo M, Etchepare M, Hirt D, et al. Nonconvulsive electrographic seizures after traumatic brain injury result in a delayed, prolonged increase in intracranial pressure and metabolic crisis. Crit Care Med. 2007;35:2830-6.

24. Reinstrup P, Stahl N, Mellergard P, Uski T, Ungerstedt U, Nordstrom CH. Intracerebral microdialysis in clinical practice: baseline values for chemical markers during wakefulness, anesthesia, and neurosurgery. Neurosurgery. 2000:47:701-9 discussion 709-10

25. Paraforou T, Paterakis K, Fountas K, Paraforos G, Chovas A, Tasiou A, et al. Cerebral perfusion pressure, microdialysis biochemistry and clinical outcome in patients with traumatic brain injury. BMC Res Notes. 2011:4:540.

26. Marcoux J, McArthur DA, Miller C, Glenn TC, Villablanca P, Martin NA, et al. Persistent metabolic crisis as measured by elevated cerebral microdialysis lactate-pyruvate ratio predicts chronic frontal lobe brain atrophy after traumatic brain injury. Crit Care Med. 2008;36:2871-7.

27. Timofeev I, Carpenter KL, Nortje J, Al-Rawi PG, O'Connell MT, Czosnyka M, et al. Cerebral extracellular chemistry and outcome following traumatic brain injury: a microdialysis study of 223 patients. Brain. 2011;134(Pt 2:484-94.

28. Nielsen TH, Olsen NV, Toft P, Nordstrom CH. Cerebral energy metabolism during mitochondrial dysfunction induced by cyanide in piglets. Acta Anaesthesiol Scand. 2013:57:793-801.

29. Molstrom S, Nielsen TH, Andersen C, Nordstrom CH, Toft P. Bedside Monitoring of Cerebral Energy State During Cardiac Surgery-A Novel Approach Utilizing Intravenous Microdialysis. J Cardiothorac Vasc Anesth. 2017;31:1166-73.

30. Grand J, Meyer ASP, Hassager C, Schmidt H, Moller JE, Kjaergaard J. Validation and Clinical Evaluation of a Method for Double-Blinded Blood Pressure Target Investigation in Intensive Care Medicine. Crit Care Med. 2018;46:1626-33.

31. Mak M, Moulaert VR, Pijls RW, Verbunt JA. Measuring outcome after cardiac arrest: construct validity of Cerebral Performance Category. Resuscitation. 2016;100:6-10.

32. Matthews EA, Magid-Bernstein J, Presciutti A, Rodriguez A, Roh D, Park $\mathrm{S}$, et al. Categorization of survival and death after cardiac arrest. Resuscitation. 2017;114:79-82.

33. Bankier AA, Fleischmann D, Windisch A, Germann P, Petritschek W, Wiesmayr MN, et al. Position of jugular oxygen saturation catheter in patients with head trauma: assessment by use of plain films. AJR Am J Roentgenol. 1995;164:437-41.

34. Nielsen N, Wetterslev J, Cronberg T, Erlinge D, Gasche Y, Hassager C, et al. Targeted temperature management at 33 degrees $C$ versus 36 degrees $C$ after cardiac arrest. N Engl J Med. 2013;369:2197-206.

35. Kirkegaard $H$, Soreide $E$, de Haas I, Pettila V, Taccone FS, Arus U, et al. Targeted Temperature Management for 48 vs 24 Hours and Neurologic Outcome After Out-of-Hospital Cardiac Arrest: A Randomized Clinical Trial. JAMA. 2017;318:341-50

36. Lorette G, Maruani A. The CONSORT statement (CONsolidated Standards Of Reporting Trials). Ann Dermatol Venereol. 2013;140:431-5.

37. Bennett JA. The Consolidated Standards of Reporting Trials (CONSORT): Guidelines for reporting randomized trials. Nurs Res. 2005;54:128-32.

38. Harris PA, Taylor R, Thielke R, Payne J, Gonzalez N, Conde JG. Research electronic data capture (REDCap)--a metadata-driven methodology and workflow process for providing translational research informatics support. J Biomed Inform. 2009:42:377-81.

\section{Ready to submit your research? Choose BMC and benefit from:}

- fast, convenient online submission

- thorough peer review by experienced researchers in your field

- rapid publication on acceptance

- support for research data, including large and complex data types

- gold Open Access which fosters wider collaboration and increased citations

- maximum visibility for your research: over $100 \mathrm{M}$ website views per year

At BMC, research is always in progress.

Learn more biomedcentral.com/submissions 